\title{
Explore3DM-A Directory and More for 3D Metrology
}

\author{
Stephen Kyle $\mathbb{D}$
}

check for updates

Citation: Kyle, S. Explore3DM-A Directory and More for 3D Metrology. Standards 2021, 1, 67-78. https:// doi.org/10.3390/standards1020007

Academic Editor: Michele Lanzetta

Received: 18 August 2021

Accepted: 22 September 2021

Published: 5 October 2021

Publisher's Note: MDPI stays neutral with regard to jurisdictional claims in published maps and institutional affiliations.

Copyright: (C) 2021 by the author. Licensee MDPI, Basel, Switzerland. This article is an open access article distributed under the terms and conditions of the Creative Commons Attribution (CC BY) license (https:/ / creativecommons.org/licenses/by/ $4.0 /)$.
Department of Civil, Environmental and Geomatic Engineering, University College London, London WC1E 6BT, UK; s.kyle@ucl.ac.uk

\begin{abstract}
Explore3DM will be an online resource to explore the diverse interests behind threedimensional measurement and three-dimensional metrology (3DM). The motivation has been the development of large-volume and portable 3D methods and systems for applications in manufacturing, an activity which has been growing for the past 40 years. However, the measurement spectrum in Explore3DM will be wider and include, for example, as-built process plant at the large-object end and X-Ray CT inspection at the small-object end. This wider spectrum will support cross-sector research at University College London (UCL) to transfer 3DM developments from one sector to another. Initially, Explore3DM will have a core directory incorporating systems manufacturers, service suppliers, research groups and disseminators of metrology knowledge. Mechanisms for solving end users' measurement tasks will add to further growth of 3DM. The resource is intended to be free to use and the directory free to join at a basic level. Premium directory sponsorship by commercial companies is expected to provide revenue to sustain and develop the resource and support 3DM development. With regard to standards, LVM and PCM systems and techniques can be difficult to assess with a standardized approach because of the highly flexible ways they can be applied. However, some standards have been developed and there is scope for more, for example in the terminology used. A dictionary will be a component of Explore3DM's future knowledge base. By presenting a first version in a centralized resource, standardized terminology will be encouraged.
\end{abstract}

Keywords: 3D metrology; large-volume metrology; portable coordinate metrology; photogrammetry

\section{Introduction}

In the past 40 years, Large-Volume Metrology (LVM) and Portable Coordinate Measurement (PCM) have grown significantly with some hundreds of systems manufacturers and service providers now in operation as a result. The technologies have been developed to provide on-site measurement, where the measuring device is brought to an object's manufacturing location. Here, it is possible to support the manufacture and assembly of large objects, such as cars, aircraft and ships, or tasks, such as 3D deformation measurement at various scales.

Technologies for portable 3D metrology have significant origins in photogrammetry and surveying, in its most accurate form known as geodesy. Their use is long established in fields such as map-making and construction, where the tools must be transportable to the locations of interest. Photogrammetry has been further developed, for example in conjunction with projected patterns to create surface scanning systems [1]. These older technologies have been complemented by more recent developments, such as the laser tracker [2] and articulated measurement arm [3]. There is now a wide range of flexible 3D measuring technologies applicable to many manufacturing situations.

However, it is essential to take a wider view of 3D measurement. Users requiring portable 3D may also make extensive use of fixed-axis coordinate measurement and should understand their relative differences, strengths and operational characteristics. The mostly optical techniques of portable 3D can measure surfaces, not what lies beneath them. To measure sub-surface form, X-ray computed tomography could be a likely option. Optical systems delivering 10 s of micrometers may work more efficiently in conjunction with 
mobile platforms located to centimeter accuracy by ultra-wideband (UWB) indoor location systems. A smartphone with appropriate software might quickly generate a 3D model of a space where several robot measuring cells are to be located.

Here are some of the related technologies relevant to a comprehensive understanding of 3D measurement and 3D metrology (3DM):

- Industrial photogrammetry and surveying As these technologies are the origin of many 3D metrology solutions, it is not surprising that they have continuing relevance, for example in the as-built modeling of the complex pipework in process plants or deformation monitoring of a bridge as traffic flows across it.

- MoCap-Motion Capture MoCap has application in medicine, e.g., for human gait analysis, and its use is particularly widespread in entertainment industries. Systems often use multi-camera, photogrammetric techniques to generate real-time modeling, typically of human movement. Accuracy may be lower than a photogrammetric system designed for metrology, but where speed and a turnkey system is required, MoCap may be a very appropriate solution.

- Optical tooling Optical tooling is an older technique where, for example, highprecision telescopes are used to align the bearings of a large driveshaft.

- CMMs-Coordinate Measuring Machines CMMs represent another older technology which maintains a strong manufacturing presence due to the high accuracies which they can deliver. They define three fixed axes in space, typically offering measuring volumes which accommodate object lengths of a few meters and are normally located in controlled environments to maintain their high accuracy. Objects to be measured must be brought to the CMM, a key difference with portable 3D.

- X-Ray CT X-Ray measurements can look below object surfaces and into object interiors, offering a 3D solution, which none of the above can offer.

Figure 1 [4-13] illustrates the spectrum of 3D measurement just described, centered on portable 3D technologies. One key element missing from this very positive development in measurement technology is an accessible, well-structured source of information which enables potential users to both understand and apply the relevant methods and systems to their own measurement challenges. A good understanding of how different techniques work, and are best applied, encourages good practice and delivers high-quality results. While automation and sophisticated algorithms have resulted in very efficient, selfcontained commercial systems, on-going requirements to specify, for example, optimally configured networks of cameras or laser trackers still require an understanding of geometric principles and operational features. Even a fixed measurement system, such as a CMM, requires a complex calibration model to generate its high accuracy and good measurement strategies to further optimize results. Just two of the many good practice guides from the UK's National Physical Laboratory illustrate some of the above with GPG080 considering aspects of PCM and GPG041 looking at CMM measurement strategy [14].

One major effort in the process of disseminating relevant LVM and PCM knowledge was initiated and continued by the Coordinate Metrology Society (CMS), an internationally active organization based in the USA. The CMS started as a special interest group of the American Society for Photogrammetry and Remote Sensing (ASPRS) in 1984 and has subsequently developed into a learned society in its own right. Nearly 40 years on, the CMS delivers [15]:

- The annual Coordinate Metrology Society Conference (CMSC) held in the US

- The Journal of the CMSC with selected peer-reviewed papers from the conference

- A Certification Programme to give recognition to expert users of PCM systems

- 3DMU—the 3D Measurement U(niversity) offering online video training 


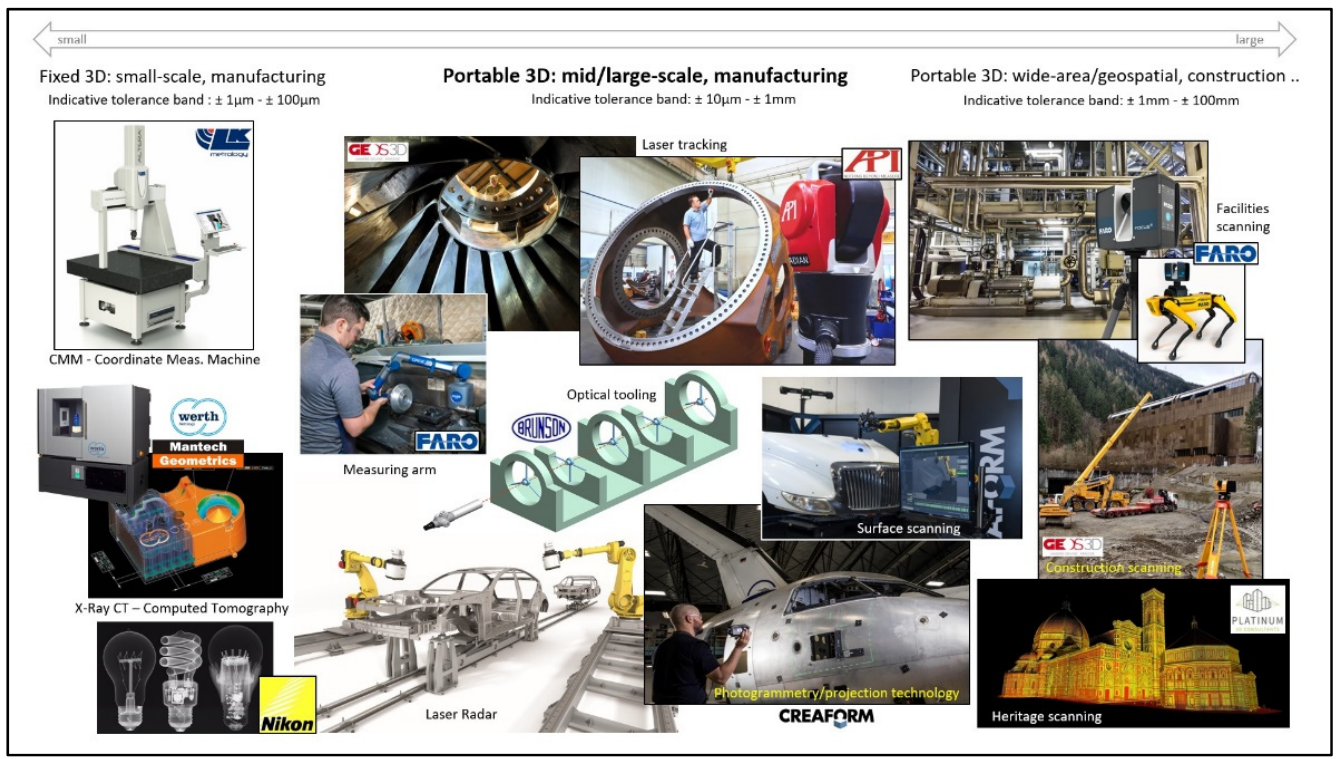

Figure 1. Spectrum of 3D measurement.

The original motivation for the specialist group was to encourage industrial users of LVM and PCM systems to present their applications and thereby assist others to adopt portable 3D metrology solutions. The conference retains this practical approach to 3DM development and the concept has been emulated to a significant extent by the 3D Metrology Conference (3DMC) [16], a relatively new annual event currently being hosted in different European countries. Despite this comprehensive effort to disseminate 3DM knowledge, much more could still be done:

1. A knowledge base incorporating:

A dictionary to provide quick access to relevant information

A library of information to complement taught and remote training

2. Problem-solving mechanisms based on applications to expand the take-up of 3DM

3. A directory encompassing the entire 3DM community to bind the above together

The presentation here builds on the directory (3), which effectively provides a snapshot of the current extent and impact of 3DM. Problem solving using application examples (2) is a snapshot of the current range of tasks which 3DM addresses. When packaged together with background knowledge (1), a unique and accessible online tool is created.

\section{Explore3DM-Background and Current Status}

Independently, and in collaboration with University College London (UCL), previous attempts have been made to create some of the components mentioned above, as well as complete solutions:

- 2010: A library of LVM on Google Docs (no longer a facility offered by Google)

- The author's structured arrangement of sample file extracts to act as a discussion tool.

- 2016: PCMA - an Academy of Portable Coordinate Measurement

- The author's private Excel interface to a large collection of instructional and learning material.

- 2017: 3DIMPact-online.com (accessed on 18 August 2021) [17] A demonstrator website from a 1-year project funded by UCL. The website requires access permission. The website demonstrates a directory, case studies and a knowledge base.

- 2018: Proposal to Engineering and Physical Sciences Research Council (EPSRC) A £500K funding proposal by UCL to create Explore3DM, an international network for 3DM. The proposal was rejected in Jan. 2019. 
- 2020: Unfunded partnership to initialize Explore3DM as a directory A directory encompassing the entire 3DM community can also act as a network. Please contact the author if any of the above is of interest.

The 2020 partnership is between the author and Marked Improvement (MI), a web development company based in the UK and Spain [18]. MI has extensive experience in content management and the development of Learning Management Systems (LMS). The development will also link with the author's research work at UCL via a new Airbus/Royal Academy of Engineering Research Chair in Large-Volume Metrology. This chair has been awarded to Stuart Robson, UCL's Professor of Photogrammetry and Laser Scanning, and will be in operation from 2020 to 2026. This funded research not only supports specific Airbus interests in LVM but also the Academy's wider interests in expanding the impact of $3 \mathrm{DM}$ via mechanisms which include metrology training and cross-sector cooperation and information exchange, e.g., between construction and manufacturing.

The website is currently under construction and has the following key objectives:

- Bring together the 3DM technology users and technology suppliers

- Include the suppliers in a directory covering the entire 3DM community, i.e., systems manufacturers, service providers, researchers and knowledge disseminators

- Make it free to access, with free basic entries in the directory

- Create sustainable revenue from premium commercial company entries

- Use a significant percentage of net revenue to support the development of 3DM Depending on available revenue, support for 3DM development will include:

- Contributing to the Academy's proposals for cross-sector knowledge transfer with activities such as:

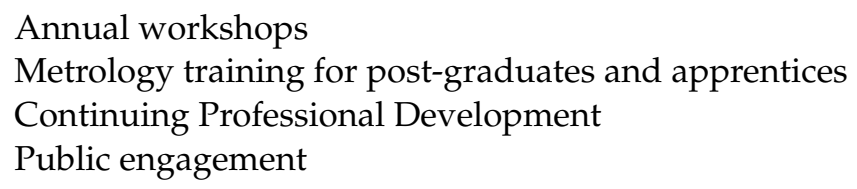

Annual workshops

Metrology training for post-graduates and apprentices

Continuing Professional Development

Public engagement

- Increasing the impact of the 3D Metrology Conference

- Improving metrology connections between the UK and Brazil

○ Already established through private work and UCL travel grants

- Outreach to bring 3DM closer to university students and professional engineers

The following sections will discuss the first areas of active development, a directory and contact exchange, then scope for further development via an applications database and metrology knowledge base, including a dictionary.

\section{The Directory of 3D Measurement and Metrology}

Over a number of years, the author has collected website links to companies, organizations and groups which are directly or indirectly relevant to the 3D metrology spectrum (Figure 1). A number of these are now organized in an Excel file with a current classification allowing for:

1. Software for data capture, analysis and display (system manufacturers)

2. Hardware for data capture (system manufacturers)

3. Services

4. Resellers/distributors of software and hardware (sales, rentals and used equipment)

5. Innovation and development (including university research groups and similar)

6. Information and events (societies, conferences, publishers, etc.)

7. Training (providers of training courses)

These are further sub-classified with 651 active organizations currently listed and already imported into the Explore3DM website under construction. Recently collected links numbering in excess of 300 are also provisionally recorded in a Word file for further evaluation prior to addition to the new website's content manager. Before website launch, all sources will be contacted for approval. 
Initiating the online resource with a directory has distinct advantages:

- Revenue-generating potential via support from listed commercial companies

- An initial mass of listed suppliers to attract a significant audience of end users

Note the current distributions by country of 544 systems and service providers, items 1-3 above, in Figures 2 and 3.

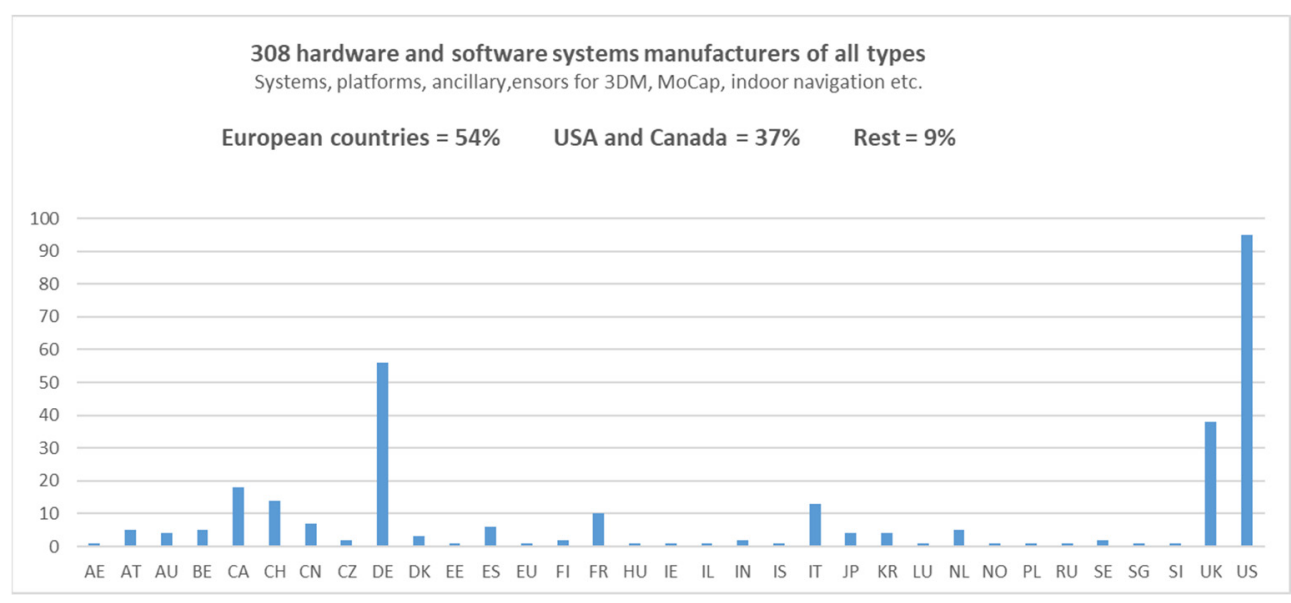

Figure 2. Distribution of systems manufacturers.

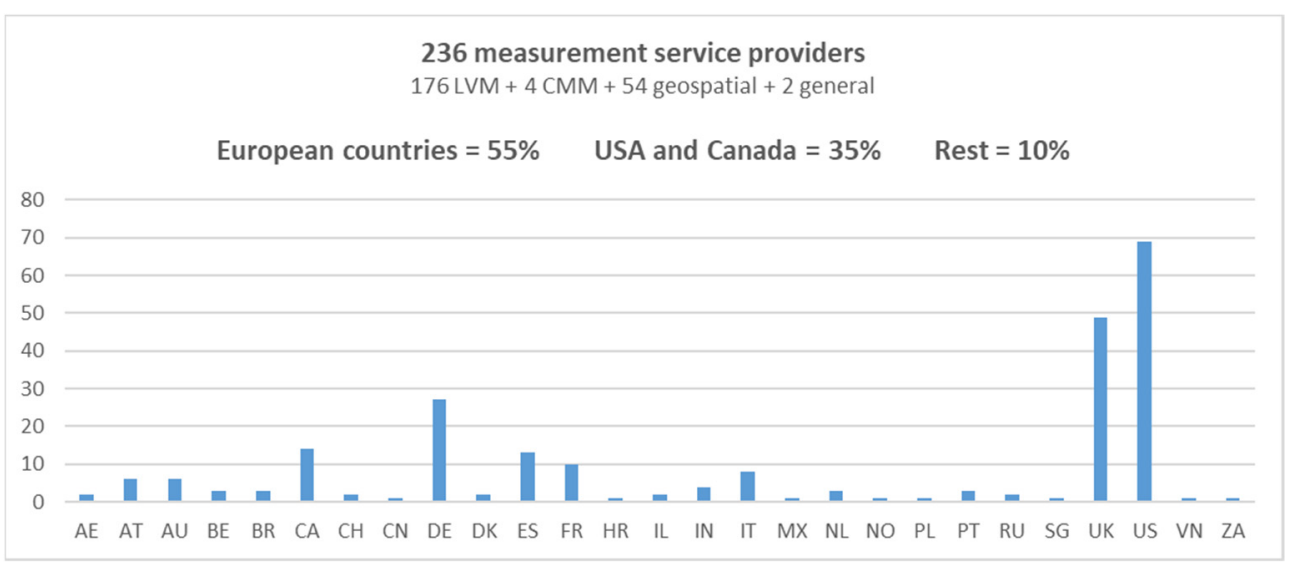

Figure 3. Distribution of service providers.

The directory will offer a free basic membership to any group or organization with relevance to the spectrum of 3D measurement and metrology. Revenue to maintain and develop the resource, and contribute to 3DM development, will be found from commercial companies willing to fund premium levels of membership. Research groups, non-profit organizations and key partners would have free premium membership in return for in-kind support, such as contributions to a knowledge base.

With suppliers and end users drawn to a contact point, there is scope to offer problem solutions.

\section{Problem Solving}

A technology directory would be particularly valuable if an end user could submit a query to it in the form "This is my measurement task. How do I solve it or develop a solution?". Then, the system should respond with, ideally, a highly focused answer of the form:

- "Systems of the following type are known to handle this task well ..."

- "This international service provider in Brazil has related experience ... "

- "These researchers in Germany have developed adaptable solutions ... " 
This would rapidly connect the user with potential sources of solution to the user's task. On one level, a user would solve a measurement challenge and suppliers would have an outlet for their goods, services or expertise. On another level, these interactions would extend the knowledge base of solutions, potentially with statistical indicators showing which are most successful. This, in turn, contributes to best practice, improved understanding and better quality control, which are key concepts for anyone concerned with standards.

\subsection{Contact Exchange-Explore3DM's "Xchange"}

The developers of Explore3DM will offer a contact eXchange (the Xchange), where users (clients) can post tasks and directory members (providers) can suggest relevant responses. This is illustrated in Figure 4.

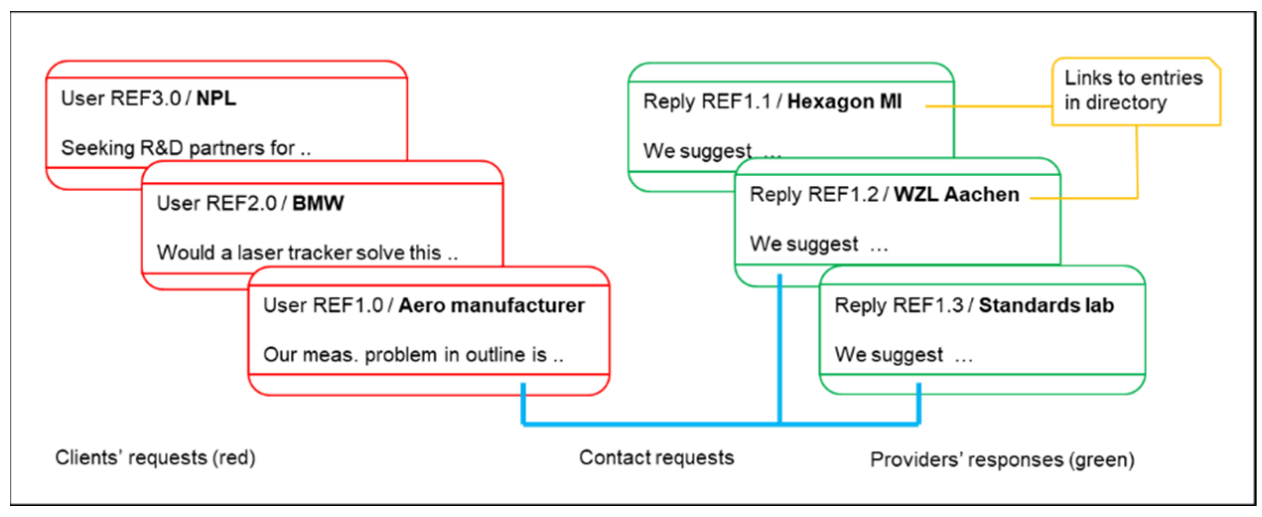

Figure 4. Solutions via the Xchange.

Not all tasks will be directly related to a measurement requirement. For example, there may be requests for partners in an upcoming research project, or advice in setting up a 3D measurement laboratory. However, many tasks will require solutions to a measurement challenge and a body of experts are an ideal source of well-considered responses. Clearly, the experts must be willing to respond to the tasks, and a classification of tasks could help direct only those of interest to particular experts.

Public viewing to some level of detail is proposed, so clients and providers may choose to name themselves generically and not with a company name, e.g., "aerospace" rather than "Boeing".

Clients might choose to make independent use of the responses or contact providers to take the discussion offline and to their own private level of detail. It is emphasized that this proposal is experimental and only through operation and modification can its effectiveness be determined.

\subsection{Applications Database and Profile Matching}

Another approach to problem solving is to build a database of known solutions which users can search for similarities to their own measurement tasks and evaluate sources of good matches.

The websites of many systems manufacturers and some service providers often provide free access to application information described as application reports, case studies, user stories or similar. The author has currently collected approximately 2000 of these documents. Thus far, some 750 can be accessed via an Excel file (Figure 5) and the remaining files will be added. The distribution of named users is shown in Figure 6. 


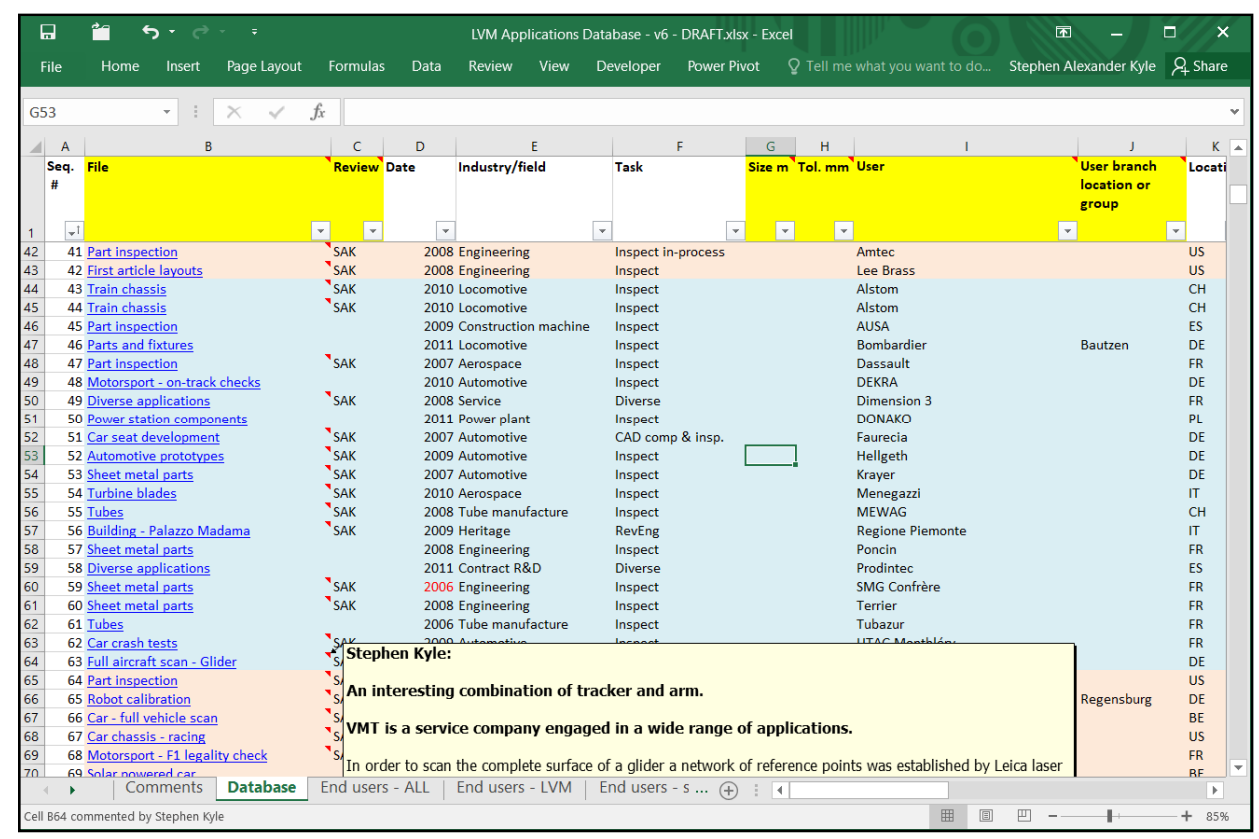

Figure 5. Author's Excel database of case studies.

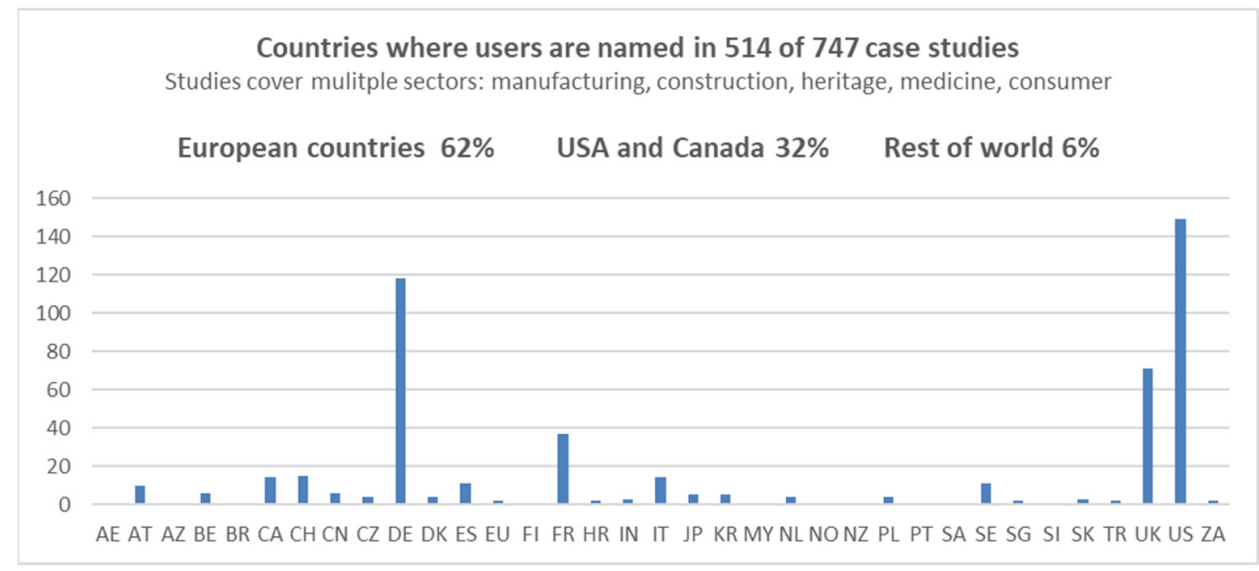

Figure 6. Distribution of named end users by country.

With this simple, in-house Excel database, it is possible to filter results by, say, company name, e.g., Siemens, and/or sector, e.g., aerospace, for a closer fit to a given measurement task. Users finding case studies similar to their own would then be able to approach the named source of the solution or look in more detail at the named technology in the solution. A potentially better search could be made if application reports contained minimum standardized information, such as:

- What is measured, i.e., an object descriptor such as "car door"

- The nature of the measurement task, e.g., surface damage or comparison with CAD

- The typical size or spread of the object or measured features, e.g., $1 \mathrm{~m}$ or $20 \mathrm{~m}$

- The tolerance met by a given solution or required by a user, e.g., $0.2 \mathrm{~mm}$ at $1^{*}$

Optionally, more detail might be added, such as:

- $\quad$ System/surface interaction, e.g., targeted

- Object properties, e.g., static or deforming

- Task operation, e.g., manual or automated

- Environment, e.g., indoors or outdoors

The above information, when specified for a particular task, constitutes a task profile appropriate either to a user's specification or a source's knowledge of an actual application 
or a solution designed for a particular class of applications. The source's information then additionally provides:

- Solution used, e.g., tracker or photogrammetry

- Documentation, e.g., link to an application report

It is proposed to evaluate profile matching as another way of finding potential solutions to the measurement tasks facing end users. Here, users' required task profiles would be matched against known application profiles. The number of returned results would depend on an acceptable level of matching set by the user. Figure 7 illustrates the process.

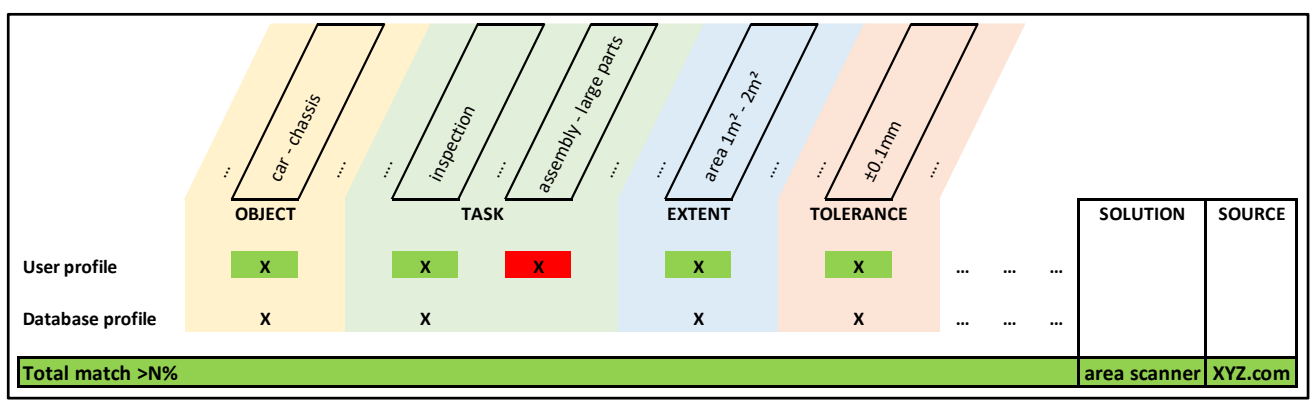

Figure 7. Task profile matching.

Note that the above process is initially based on data extracted from publicly accessible information but the additional supply of that information, such as an application report in PDF format, would require the agreement of the source.

\section{Knowledge Base-A Dictionary and Library}

Some basic introductory information would be highly appropriate as part of Explore3DM. It would help newcomers to find their way around the various technologies and applications of 3DM so that they could, at an early stage, make effective use of the directory and problem-solving tools on offer. It would also deliver a suitable response to the Royal Academy of Engineering's requirement for public engagement. Once in place, further information could be added to establish a multi-media library and comprehensive knowledgebase of 3DM.

Library information also has potential to connect with the directory, as another way of promoting resources which technology suppliers can provide.

Figure 1 is related to an existing multi-slideshow overview of 3DM, which contains a slide on calibration, adjustment and alignment, similar to Figure 8 . Here, one image points to systems manufacturer FARO [7], showing a laser tracker solution, and one to research group i3mainz [19], showing a photogrammetric solution. The slide has been created by UCL's 3DIMPact Group [20]. Users of the directory might, for example, click on the logos for more information on the organizations behind the information.

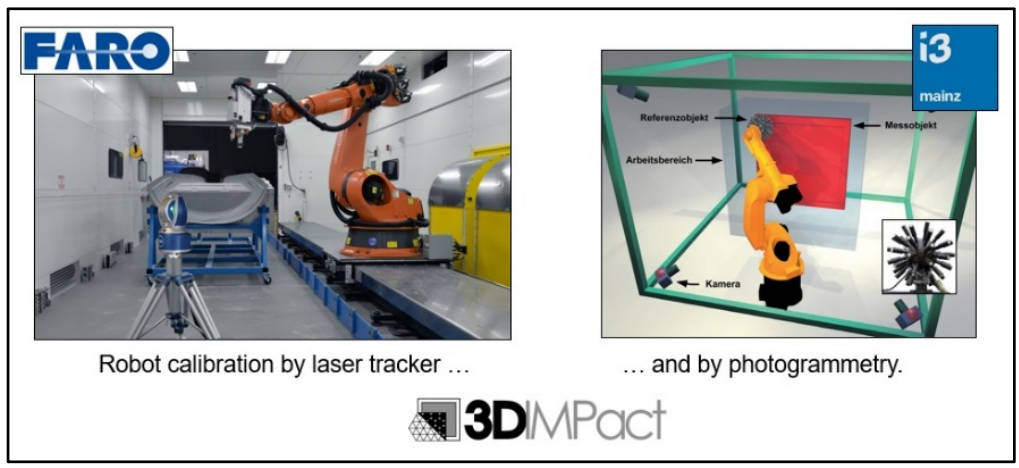

Figure 8. Illustrative introduction to calibration and alignment. 


\section{Dictionary —An Opportunity for Standardized Terminology}

As an alternative to a structured presentation, a dictionary offers the ability to directly look up terms and, ideally, their context and connection to other terms.

The author's prototype dictionary illustrates some features of interest. Figure $9 \mathrm{a}$ shows an example from the Axyz Dictionary of 3D Metrology, created as a WinHELP file by the author in 2000 for Leica Geosystems. The example explains "corner cube" with optionally available additional information displayed in separate windows (diagram of the reflection process, mathematics of reflection). Figure $9 \mathrm{~b}$ is a later Word version of the dictionary illustrating a definition of "jointed arm CMM" with illustrations from FARO, which could, again, link to the FARO entry in the directory.

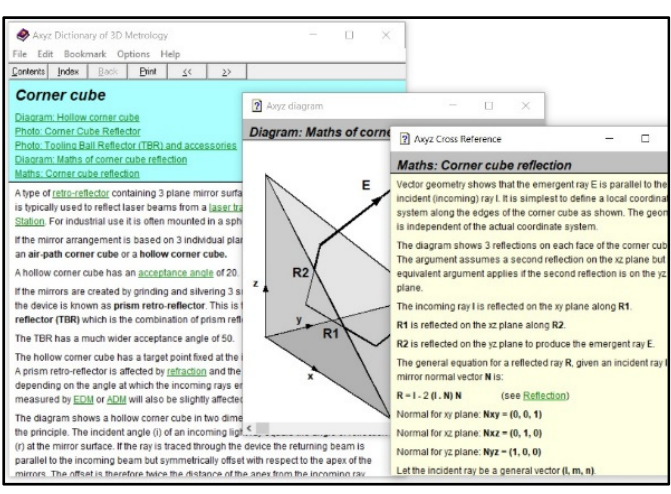

(a)

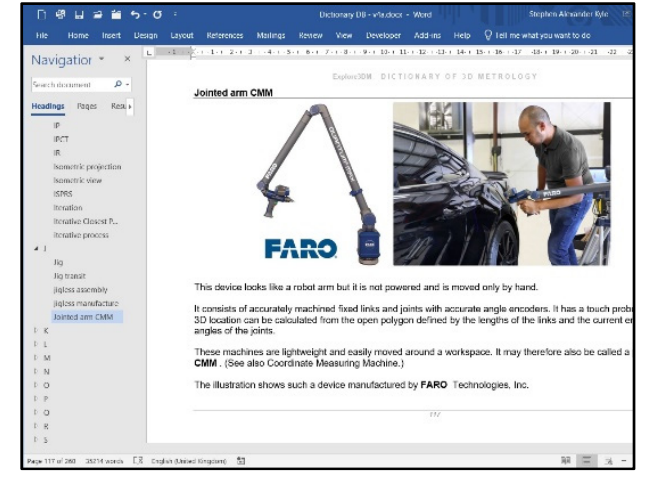

(b)

Figure 9. Prototype dictionary in two formats; (a) WinHELP file (2000), (b) WORD file (2021).

Explore3DM is not primarily about developing standards, but it can clearly act as a focal point for encouraging the use and further development of standards. One aspect of 3DM lacking in standardization is terminology in the proposed dictionary. Here, a few examples will highlight some areas where usage is inconsistent and alternative terms describe the same item. The examples all have relevance to 3DM, and thus, appear in the author's 1000-term draft dictionary.

- In English, should there be a preferred spelling option: coordinate or co-ordinate?

- Drones are useful mobile platforms in 3D measurement, even indoors. The term "drone" is in everyday use but is it an acceptable technical term or should we only use a term such as Unmanned Aerial Vehicle (UAV)? Or should that be UAS, where "S" is for system? Or both?

- Which term is preferable: "CMM arm" or "Articulated Arm Coordinate Measuring Machine (AACMM)" as used in the standard ASME B89.4.22?

- $\quad$ Are Laser Radar and LiDAR the same thing?

- Does Frequency Scanning Interferometry (FSI) do the same as "chirping" in Laser Radar, also called Coherent Laser Radar (CLR)? If so, is there an overlap in terminology which should be resolved or are different terms required?

- A "stereo camera" is sometimes identified as a "3D camera". However, the latter might be a range/imaging camera which operates in a different way from the dualimaging stereo camera. Both would seem to deserve a separate definition in order to distinguish between them.

- There are manufacturers of "optical CMMs" which incorporate a moving probe or scanner, together with an "optical tracker" which has three cylindrical lenses fixed in a single housing. Each generates a line image of LED targets on the probe, locating them spatially by the intersection of three planes. Should this have a more specific name than "optical tracker", e.g., "triplanar camera" or "3-line imager"?

As a final example, it could be expected that angle conventions in 3D geometry are well established but usage can be mixed. 
- The term "vertical angle" is sometimes used for an angle in the (nominally) vertical plane, which is zero when pointing not horizontally but along the zenith, which is vertically up. In this case, the author prefers the term "zenith angle", with "vertical angle" reserved for an angle which is zero when indicating a horizontal direction.

- There is a similar situation with horizontal angles where an angle in the XY plane zeroed in the $\mathrm{X}$ direction is then $90^{\circ}$ on the $\mathrm{Y}$ axis, and hence, positive anticlockwise. However, this angle is often also named an "azimuth angle", which, in surveying and mapmaking, is an angle zeroed when pointing north and $90^{\circ}$ when pointing east, and hence, positive clockwise.

This mixed terminology can be seen, for example, in [21], which is perfectly clear in its presentation but is given here as an example of mixed usage. Confusions arise, however, when presentations are not so clear, hence justifying some degree of standardization.

\section{Cross-Sector Engagement in LVM}

LVM and PCM incorporate highly scalable measurement techniques and are ideal for exploring and implementing cross-sector exchanges of 3D measurement and metrology solutions. This is an aspect to UCL's research work, which is funded by the Royal Academy of Engineering and which will benefit from the development of Explore3DM. Existing examples will illustrate the potential here. These examples show measurement at mixed scales and the application of closely related techniques in different sectors.

Very mixed scales are evident in particle accelerator alignment where beams of subatomic particles are guided on linear and circular paths, for example bringing opposing beams into collision in order to gain insights into the structure of matter. One of the new accelerators, the Brazilian Synchrotron Light Laboratory known as Sirius, is located in a circular tunnel about half a kilometer in circumference. Within this large civil engineering construction, magnets must be aligned to $10 \mathrm{~s}$ of micrometers in order to maintain and direct the particle beam. A measurement network covering the entire ring achieved submillimeter uncertainty, thus demonstrating alignment accuracies at construction scales. See the video by Geraissate, directly viewable on the 3DMC website or downloadable from the 3DMC archives [16]. A number of such accelerator alignment groups can be found in the directory.

The directory also lists service companies which offer both engineering surveying services, such as as-built modeling of process plant, together with industrial metrology services, such as precision alignment. The website of Heinz Meschke in Germany is a good illustration of this. See "Ingenieurvermessung" and "Industrievermessung" [22].

Finally, note how American company Capture3D reports on the use of system manufacturer GOM's 3D surface scanner, ATOS, for cultural heritage recording of a bronze sculpture [23] and for digitizing the mold of a protective mask [24]; heritage on the one hand, manufacturing on the other.

\section{Further Information and Future Development}

This review in the launch issue of "Standards" was based on an online presentation of the current status of Explore3DM delivered by the Midlands Centre for DataDriven Metrology [25]. This center was launched in November 2020 and is a multisite collaboration between the University of Nottingham, Loughborough University, and Coventry University.

With regard to future development, there are many opportunities to consider, some of which will not be apparent until Explore3DM is in operation. One area of particular interest relates to the applications database, a potential source of problem solutions. A first-stage development would be a standardized approach to defining task profiles in order to improve the search functionality. As this database expands, better matching techniques may be possible, e.g., using artificial intelligence to analyze the task database. 


\section{Conclusions}

Explore3DM is a novel and developing resource which will initially deliver a comprehensive directory of 3D metrology suppliers and a contact exchange to help end users identify suppliers who can assist with their metrology tasks. In operation, it should support the development of 3D metrology by giving the entire 3D metrology community, both commercial and non-profit, a location to showcase their products, services and skills and make new connections.

As it develops, it will encourage good practice and, in some cases, standardization where there are current limitations.

Funding: UCL funding to develop the demonstrator website at 3DIMPact-online.com (accessed 18 August 2021) was obtained from the EPSRC Impact Accelerator Account (IAA) under grant reference $\mathrm{EP} / \mathrm{K} 503745 / 1$.

Acknowledgments: UCL's current research work in Large-Volume Metrology, funded by the Royal Academy of Engineering, provides a valuable focus on LVM applications addressed by Explore3DM such as cross-sector technology transfer. This research is funded by grant "RAEng/Airbus Chair in Large Volume Metrology RCSRF1920 $10 \backslash 35^{\prime \prime}$.

Conflicts of Interest: The author declares no conflict of interest.

\section{References}

1. Brown, D.; Laurie, J.-F. Portable Metrology. In Handbook of Optical Dimensional Metrology; Harding, K., Ed.; CRC Press: Boca Raton, FL, USA, 2013; pp. 265-286.

2. Sandwith, S.; Kyle, S. Laser Tracking Systems. In Handbook of Optical Dimensional Metrology; Harding, K., Ed.; CRC Press: Boca Raton, FL, USA, 2013; pp. 93-155.

3. The Origins of the Articulated Arm CMM. Available online: https://www.hexagonmi.com/solutions/technical-resources/ metrology-101/the-origins-of-the-articulated-arm-cmm (accessed on 18 August 2021).

4. API-Automated Precision, Inc. Available online: www.apimetrology.com/ (accessed on 2 September 2021).

5. Brunson. Available online: www.brunson.us (accessed on 2 September 2021).

6. Creaform (Ametek). Available online: www.creaform3d.com/en (accessed on 2 September 2021).

7. FARO. Available online: www.faro.com (accessed on 2 September 2021).

8. GEOS3D. Available online: www.geos3d.com (accessed on 2 September 2021).

9. LK Metrology. Available online: www.lkmetrology.com/lk_newsite/ (accessed on 2 September 2021).

10. Mantech Geometrics. Available online: www.mantechgeometrics.co.uk (accessed on 2 September 2021).

11. Nikon Metrology. Available online: www.nikonmetrology.com (accessed on 2 September 2021).

12. Platinum 3D Consultants. Available online: www.platinum-3d.com (accessed on 2 September 2021).

13. Werth Messtechnik. Available online: www.werth-metrology.com (accessed on 2 September 2021).

14. All Good Practice Guides. Available online: www.npl.co.uk/resources/gpgs/all-gpgs (accessed on 18 August 2021).

15. CMSC. Available online: https://www.cmsc.org/ (accessed on 18 August 2021).

16. 3DMC. Available online: https://3dmc.events/ (accessed on 18 August 2021).

17. 3DIMPact-Online. Available online: http://www.3dimpact-online.com/ (accessed on 18 August 2021).

18. Marked Improvement. Available online: www.markedimprovement.co.uk (accessed on 6 September 2021).

19. Boochs, F.; Schütze, R.; Raab, C.; Wirth, H.; Meier, J. A flexible multi-camera system for precise tracking of moving effectors. In Robotics and Applications; Cambridge, MA, USA, 2009. Available online: https:/ /www.semanticscholar.org/paper/A-FLEXIBLEMULTI-CAMERA-SYSTEM-FOR-PRECISE-TRACKING-Boochs-Sch\%C3\%BCtze/badd07fea53de9772c39247a49100b34bf598 c1a (accessed on 18 August 2021).

20. 3DIMPact Group at UCL. Available online: www.ucl.ac.uk/civil-environmental-geomatic-engineering/research/groups-centresand-sections/3dimpact (accessed on 6 September 2021).

21. Wang, L.; Muralikrishnan, B.; Rachakonda, P.; Sawyer, D. Determining geometric error model parameters of a terrestrial laser scanner through two-face, length-consistency and network methods. Meas. Sci. Technol. 2017, 28, 065016. [CrossRef] [PubMed]

22. Heinz Meschke Ingenieurvermessung. Available online: https:/ /www.vermessung-meschke.de/industrievermessung/ (accessed on 18 August 2021).

23. Digitizing of the Ancient Bronze Sculpture of Apoxyomenos. Available online: https://www.capture3d.com/case-studies/ entertainment-arts/croatian-conservation-institute-digitizing-of-the-ancient-bronze-sculpture-of-apoxyomenos (accessed on 18 August 2021). 
24. 3D Scanning of a Die-Cast Mold for a Protective Mask. Available online: https://www.capture3d.com/case-studies/injectionmold-plastics/3d-scanning-of-a-die-cast-mold-for-a-protective-mask (accessed on 18 August 2021).

25. MCDDM: Monthly Seminar-July 2021. Available online: www.mcddm.ac.uk/events/past-event-recordings/past-events-home. aspx (accessed on 3 September 2021). 